Hydrol. Earth Syst. Sci. Discuss.,

https://doi.org/10.5194/hess-2018-452-AC1, 2019

(C) Author(s) 2019. This work is distributed under

the Creative Commons Attribution 4.0 License.
Interactive comment on "Reconstructing the Salgar 2015 Flash Flood Using Radar Retrievals and a Conceptual Modeling Framework: A Basis for a Better Flood Generating Mechanisms Discrimination” by Nicolás Velásquez et al.

Nicolás Velásquez et al.

nicolas.velasquezgiron@gmail.com

Received and published: 12 January 2019

article [utf8]inputenc [top $=1 \mathrm{in}$, bottom $=1.25 \mathrm{in}$, left=1.25in, right=1.25in]geometry

natbib graphicx xcolor tabularx graphicx adjustbox 


\section{Response to Reviewer 1}

January 12, 2019

Manuscript title: Reconstructing the Salgar 2015 Flash Flood Using Radar Retrievals and a Conceptual Modeling Framework: A Basis for a Better Flood Generating Mechanisms Discrimination

Authors: Nicolás Velásquez, Carlos D. Hoyos, Jaime I. Vélez, and Esneider Zapata

We sincerely thank the anonymous reviewer \#1 and Dr. Eric Gaume for their careful and thoughtful reviews. We have taken their considerations into account and have responded to their concerns both in the paragraphs below and within the manuscript. We feel the current manuscript is indeed better thanks to the reviewer comments. Below, the reviewer comments are in black and our comments are in "blue". 


\section{Response to Anonymous Reviewer \#1 General Comments}

We really appreciate the detailed work by anonymous reviewer \#1. We now recognize the point raised by reviewer \#1 about the potential lack of clarity of the paper having two main objectives. We decided to focus on the process understanding goal and we rewrote several paragraphs of the paper trying to address the clarity issue. More weight is thus given to the process understanding goal, and the forecast issue is only mentioned directly in the discussion section; we believe mentioning the forecast issue makes the paper stronger from an applied point of view.

Also, in the current version of the manuscript, there is a detailed explanation regarding the model set-up, parameter selection, and model calibration, a point raised by reviewer \#1. As described in the current version of the manuscript, some parameters are indeed from literature, and others from model calibration from other basins in the region with similar properties, and from sensitivity analysis.

We fully agree with reviewer $\# 1$ in that the conclusions derived from modeling results have limitations and those were not fully acknowledged nor discussed properly in the original manuscript. This issue was also raised by reviewer \#2 (Dr. Eric Gaume). The current manuscript addresses the potential uncertainty of the modeling results in the discussion section, including a comparison with existing literature. Due to the data scarcity in the region there is an inherent impossibility to validate the model. To address this issue we included a sensitivity analysis showing the main conclusions regarding process understanding are the same for different model calibrations using parameter values within physically plausible ranges. Reviewer 1 also points to the fact that the equifinality issue is not discussed in the original manuscript; we now address this issue in the results and discussion section based on the sensitivity analysis, also highlighting the fact that the original model calibration not only considered peak discharge but also the spatial distribution of the flood plains and landslides.

Several changes were made to the manuscript to improve the description of the meth- 


\section{Response to Specific Comments}

1 Abstract: quite long : the first 8 lines might be removed

The abstract has been modified accordingly. It was also shortened and partly rewritten to focus on the process understanding goal.

2 Introduction: the introduction is too long. The bibliography done is significant but irregular and sometimes out of the scope. I would suggest to follow the plan : i) Flash flood: definition, hazard risk; ii) Catchment and meteorological features controlling the flash floods AND landslides processing; iii) Flash flood in Colombia, the specific Salgar flash flood events; IV) ssue : Flood forecast and modelling with scarce data; V)Objectives of the paper, method, plan.

The introduction has been shortened considerably following reviewer 1 general plan and the detailed comments in the 3 . We really appreciate reviewer 1 suggestions regarding the Introduction.

3 Introduction: I would suggest the following modifications:

3.1 from page 2, line 52 to page 4, line 106 : the literature should be more concise and be reduced up to 2 or 3 paragraphs. The plan of the statements declared page 2, line $48-52$ should give guidelines for the organization of the paragraphs.

The first paragraph has also been shortened, and the lines 52 to 106 were reorganized and shortened.

3.2 Page 2, line 53 - 55 and page 4, line 108 - 116 : to my point of view, those lines are out of the scope and could be removed, making clearer the introduction. 
Lines 53-55 and lines 108-116 have been removed from the introduction.

3.3 Page 125-129: are the figure related to Colombia alone or the Caribbeans and the South of America? As the paragraph starts with a description of Colombia, I suggest to give statistics related to the country itself, in order to avoid confusion.

The statistics are only for Colombia. This issue has been clarified in the text.

3.4 Page 5, line 131-148: the full description of the events should be located in the 'Data and area of the Study'. Here a succinct description with argument for showing the interests of that particular study case are expected. As example: 'The paper focuses of two consecutive rainfall storms that took place in May 15th and May 18th 2015 in La Liboriana upstream, a 56 km2 catchment located in the Western range of the Colombian Andes. The resulting flash floods dramatically affected the local population, leading to more than 100 casualties, and to an estimated total cost of 36000 millions Colombian pesos (ấij 12.5 millions \$, considering the 2018 rate) for infrastructure reconstruction and community supports. In spite of the data scarcity, including discharge measurements, that two successive events provide an interesting study case for assessing the flash flood mechanism processes with contrasted rainfall forcing distribution and soil moisture conditions.'

We modified the paragraph following closely referee \#1 comments.

3.5 Page 5, line 161: At the end of the paragraph, it should be specified which method is chosen here (the third one, I guess).

Yes, we followed the third approach. This is now explicitely mentioned in the manuscript. We also deleted most of lines 163-174 for clarity.

3.6 Page 6, line 175 - 191: here again the description of the method should be more concise and detail has to given in the third section. I suggest the following modifications: The methodology followed in this study makes use of a conceptual modeling framework that includes a hydrological model (Vélez

Printer-friendly version

Discussion paper 
(2001) and Francés et al. (2007)), a shallow land-slide sub-model (Aristizábal et al., 2016), and a hydraulic sub-model (HydroFlash). The hydroHESSD logical model includes virtual tracers to explore separately the role of runoff and subsurface flow, as well as the relative importance of convective and stratiform precipitation in flash flood generation. A comparison between the results from both sub-models and the observed landslides scars and flooded spots helps to evaluate the overall skill of the proposed methodology.

In this case we also modified the paragraph following closely referee \#1 comments.

4 Section 2: Study site and data

4.1 To clarify the structure, two subsections might be established: 2.1 = Catchment description / properties -2.2 = Flash flood event observations.

We modified the document to include the subsections suggested by referee $\# 1$.

4.2 Figure 2: Please indicate the size resolution of cells used when calculating HAND.

HAND values where estimated using the same resolution of the DEM (12.7 $\mathrm{m})$. We included this information in the caption of the Figure 2.

4.3 Pages 8-9, lines 218 - 245 : Maps of the slopes, vegetation cover (even roughly designed), and soil properties would provide a better illustration of the description.

Printer-friendly version

We have added the slope map as part of Figure 2. Soil properties and vegetation cover maps are part of a national cartography with a scale of

Discussion paper

C6

Interactive

comment

Discussion paper 
$1: 400.000$; at this scale there is almost no variability in the watershed. Nevertheless, we added Figure 1 showing land use in different regions of the watershed from a 2012 satellite image. According to zoom 1, the vegetation is denser in the upper region of the basin, with the presence of grass. Downstream (zoom 2), it is evident the presence of crops among forest and grass. Near the middle of the basin (zoom 3), the presence of crops is more notorious and human settlements and roads start to appear. In zoom 4, the first affected urban area from upstream to downstream, it is also possible to see a marked presence of crops and some forest patches. Finally, zoom 5 shows the main urban area of Salgar surrounded by crops, grass and an important loss of forest coverage. We modified Lines 229-234 in the original manuscript, accordingly.

4.4 Figure 3: The information content of this figure is quite repetitive with the figure 2. I suggest to remove it.

We agree with referee \# 1 and we have removed the figure. Slope information was added to new Figure 2 in the new manuscript.

4.5 Page 9, lines $235-245$ : soil information. The lines 236 and 240 are not of interest and could be removed. In contrast, some information about how Osorio (2008) obtained the data could be relevant.

Lines 236 and 240 were removed following referee \# 1 suggestions. Also, we included some information about how (?) obtained the information.

4.6 From page 9, line 246 to page 10, line 255. The description of the data used for validation of the models should be more detailed in this section. Specially we should find the following information: How was determine the maximum flood discharge, the landslides and flood plain areas? Which data were used? Which method was used to extracts area contours as

Printer-friendly version

Discussion paper
Interactive

comment 


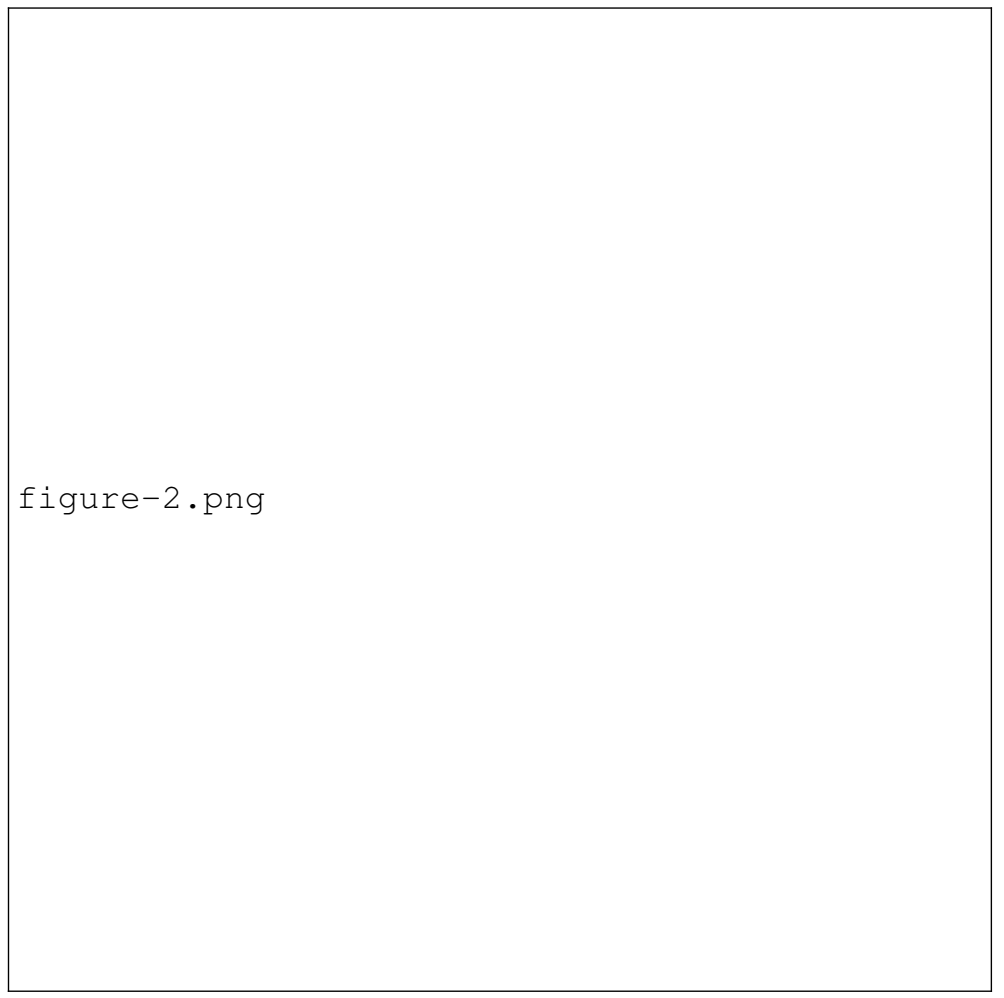

HESSD

Interactive

comment

Fig. 1. Land use in different regions of La Liboriana watershed from a 2012 satellite image.

showed in the results? Which uncertainty?

Printer-friendly version

We conducted a field campaign a few days after the flash event to assess cross-section geometry along the main channel in different sites, including at the outlet of the basin. During the campaign, we measured sectional 
distances and surface water speeds at different points of the streamflow. We also identified traditional post-event terrain, land-cover, vegetation and infrastructure markers to assess the high-water mark associated with the peak of the flash flood. Figure 4 presents the selected cross-section used for the estimation of the maximum discharge during the flash flood given its geometrical and hydraulic regularity. The section has a rectangular shape, $4.6 \mathrm{~m}$ wide and a height of $5 \mathrm{~m}$ for a total area of about $23 \mathrm{~m}^{2}$. A visual inspection of the flooded house around this section, located 4-5 $m$ away from the channel, reveals the presence of mud marks on the walls with heights varying between 0.5 and $1.2 \mathrm{~m}$ (see Figure 4). The area of the section plus the flooded area during the event was estimated to be approximately $37 \mathrm{~m}^{2}$. During the campaign we measured surface speeds in the channel oscillating between 2 and $3 \mathrm{~ms}^{-1}$. In instrumented basins in the region, with similar characteristics in terms of area and slopes, we have recorded peak flow surface water speeds oscillating between 5 and $7 \mathrm{~ms}^{-1}$. By assuming an area of $37 \mathrm{~m}^{2}$ and the mentioned surface speeds, we estimate that the observed flash flood peak flow may have been between 185 and $222 \mathrm{~m}^{3} \mathrm{~s}^{-1}$. The timing of the peak flow is also important information. Local authorities reported that the flash flood impacted the urban area around 02:00 A.M.

There is also relevant aerial information before and after the occurrence of the event. During 2012, the Department of Antioquia conducted a detailed aerial survey of the Salgar municipality, and few days after the event Google displayed publically high detailed satellite images of the same region. The contrast between both products provides information about flooded areas and landslide locations. Field campaign estimates and aerial imagery are central to validate the results obtained from the proposed models. 
5.1 This section in the current form is difficult to follow, as the description jumps from one model to another and finally comes back at the first mentioned one. I would suggest to reorganize this section in order to follow the method firstly announced in the introduction : 3.1 hydrological model description (that have to include hydrological scheme modification and the tracers implementation within the hydrological model); 3.2 landslide model description; 3.3 floodplain model description. Rainfall data processing has to be presented in Section 2.

We rearranged the section according to reviewer 1 suggestions.

5.2 The methodology section should strictly provide the method description, and the underlying assumptions made. Any argument to justify the objective of the study should be remove from this section (as example: lines $293-302$; 316 - 319; 326 - 332; 345 - 347; 375- 382).

We followed the suggestion of reviewer and, after rearranging and rewriting some parts of the methodology section, the lines mentioned were removed.

5.3 The Figure 5 should be the key figure offering a clear visual description of the overall method applied in this manuscript. To my understanding of the achieved work, this diagram should rather have three levels (top to down of the diagram): i) two panels showing the data inputs of the hydrological models, i.e. the DEM, the radar-based QPE, and the radar-based QPE processing according to Steiner, 1995); ii) one panel showing the hydrological model (Francés, 2007); iii) three panels showing the 3 results of the overall methods : the discharge simulation with water origins and paths information, the landslides submodel, and finally the flood plain assessment.

We changed Figure 5 according to the reviewer suggestions and we think it offers now a clearer picture of the work. See Figure 2 in this document. 


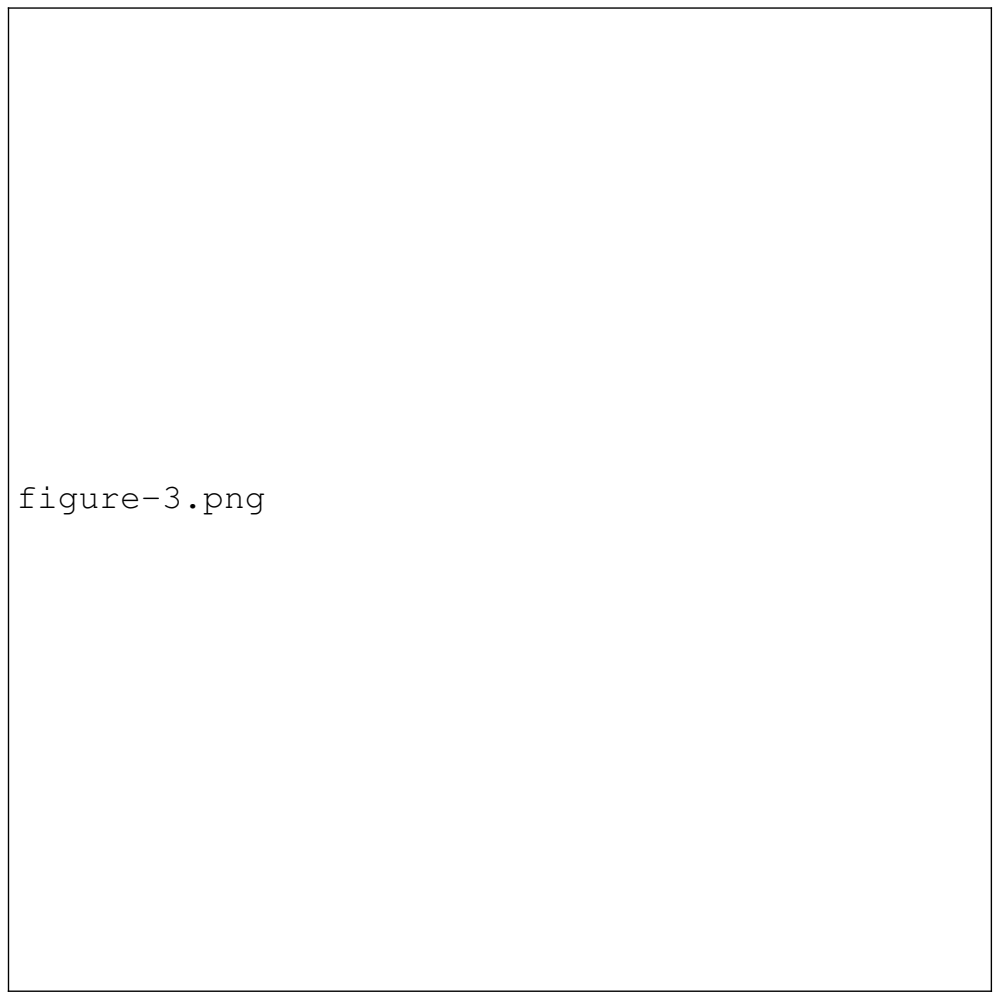

HESSD

Interactive

comment

Fig. 2. New methodology figure.

5.4 Descriptions of the models : it should useful to clarify through tables, which param- eter/information is required, how the parameters were set up (calibration ? literature value, observed data?), and whether they are spatially distributed or uniform.

The hydrologic model requires a total of ten parameters. Table 1 includes 
Gravitational storage $\mathrm{Hg}$ [mm] 134 As a function of the slope

Evaporation rate Etr $[\mathrm{mm} / \mathrm{s}] \quad 0.10 .01$ As a function of the DEM

Infiltration rate $\mathrm{ks}[\mathrm{mm} / \mathrm{s}] 2.70 .0012$ Lumped

Percolation rate $\mathrm{kp}[\mathrm{mm} / \mathrm{s}] \quad 0.80 .00012$ Lumped

Interactive

System losess $\mathrm{Kf}[\mathrm{mm} / \mathrm{s}] \quad 00.0$ Lumped

Surface speed vr $[\mathrm{m} / \mathrm{s}] \quad 0.5 \quad 6.4$ As a function of the slope and storage

Subsurface speed vs $[\mathrm{m} / \mathrm{s}] 17.1$ As a function of the slope, $\mathrm{Hg}$ and storage

Subterranean speed $\mathrm{vb}[\mathrm{m} / \mathrm{s}] \quad 0.50 .000095$ Lumped

Channel speed vc $[\mathrm{m} / \mathrm{s}] 10.95$ As a function of the slope, acumulated area, and storage

Table 1. Hydrologic model parameters.

all the parameters used in the model. Due to the lack of detailed information in the region, parameters such as the infiltration and percolation rates are assumed as constant in all the basin. There are also parameters that vary as a function of geomorphological characteristics of the basin such as the elevation and slope. According to (?), during the calibration process, each physical parameter is scaled by a constant value. Table 1 includes the mean value for all the parameters used in the model and the scalar value parameter adjusted during the calibration.

5.5 Flash flood model (I would say flood plain model) : I personally have some trouble to understand the method applied. There is no reference or explanation of the assumptions behind the equations $(10,11,12)$. In addition, several symbols are not defined: $\mathrm{Cj}, \mathrm{S} \mathrm{Cmax}$, and others are detailed $(\mathrm{Si}, 0)$ while not used in the equations, suggesting that an equation is missing in the manuscript.

We re-wrote the methodology for the flash flood model and made sure all the terms in the equations are properly described.. 
5.6 Page 16, line 423: About the hydrological runoff modification : why did you proceed to this modification? Is an adaptation of the model to the studied catchment?

Before our implementation, there were two major versions of the model. There is the SHIA (Simulación Hidrológia Abierta) version presented by (?) in his PhD thesis dissertation, and the TETIS version presented by ?. Compared to TETIS, SHIA allows the modeler to use linear and no-lineal approximations for the estimation of the horizontal speeds. From the literature (??) there is evidence that non-linear approximations increase modeling performance at detailed spatiotemporal scales. For this reason, we implemented the SHIA approximation proposed by (?).

\section{Section 4 : Results}

6.1 In the current manuscript's form, the section 4 contains only one subsection, which doesn't make sense. One logical plan considering the objective of the paper should be: 4.1 Validation of the hydrological model; 4.2 Description of the flash flood mechanism processing; 4.3 Assessment of the landslide simulation; 4.4 Assessment of the flood plain simulation (or 4.3 and 4.4 could be also merged).

We subdivided the results section into 3 sub-sections: 4.1. Hydrologic model validation and sensitivity analysis, 4.2. Flash flood processes, and 4.3. Landslide and flood simulations

6.2 The description of the flash flood mechanism processing are really detailed while the other results are summary explained : to be more balance

We have balanced the different subsections in the results section. We have also added results from a sensitivity analysis of the hydrologic simulation.

6.3 fig 12: if I understand well, the figure 12 shows spatial distribution characteristics of the precipitation over the catchment, but it is not a result of

Printer-friendly version

Discussion paper
Interactive

comment C13 
the hydrological simulation. This figure 12 should be better located in the section 2 .

Former Figure 7, only dealing with rainfall features, was moved to section 2. Figure 12 do show the spatial distribution of the characteristics of the precipitation over the catchment. The Figure is not a result of the simulation, nevertheless, along with Figure 13 explains relations between observed and simulated processes and we decided to keep it in the results section.

7 Section 5: the discussion deals only with the description of the hydrological simulation. The discussions on the landslide model and on the flood area assessment are clearly missing.

In the interest of the overall length, and as announced in the last paragraph of the introduction, we decided to maintain the discussion section focused on the hydrological simulation results, as well as the processes generating the Salgar flash flood, and the importance of the spatio-temporal variability of rainfall.

\section{Response to Technical Comments}

T1 page 6, line 199: section 5

Section corrected.

T2 page 7: reword the section 2 'Study site and data'

The title was modified following the comment.

T3 Figure 2: remove 'Las margaritas village' as it doesn't appear anywhere else in the text.

Printer-friendly version

The reference to 'Las Margaritas' was removed. 
T4 Page 9, line 246: please write 'carried out for assessing' instead of 'instrumental in obtaining'

The section was rewritten and the sentence was modified.

T5 Page 9, line 246: specify 'after the second flash event'

Interactive

The line was edited.

comment

T6 Page 10, line 268 : write 'minute' instead of 'min'

The line was changed.

T7 Page 10, lines $269-274$. The sentences were twice written. Please, remove the duplicate.

The paragraph was corrected.

T8 From page 9 line 246 to page 11 line 274. The two paragraphs give i) the available observations used in the study for assessing the hydrological model and submodels and ii) the rainfall input data used to force the hydrological model. I would change the order to respect the chronological use of the data. In addition, I would suggest to add a table summarizing the observation information available and used to validate the models.

The section was modified and restructured. Also, a summary table was included to provide a quick reference for the reader.

T9 Figure 5: we can't read any legend of the hydrological model, please uniform the size of the annotation in this figure.

Printer-friendly version

The Figure was edited; please see Figure 2.

Discussion paper

T10 Page 11, line 285: what is Ri? 
Radar data QPE rainfall estimations 2015-05-17 to 2015-05-18 Hydrological model runs. Rainfall characterization and event analysis.

Field campaign Maximum streamflow estimation trough visual inspection 2015-05-20 Hydrological model comparison for indirect validation.

Satellite imagery Real visual compositions of Digital Globe CNESimagery 2015-05 after the event

Flash flood model validation, shallow land-slides model validation, and comparison with pre-event conditions.

Aerial photos Aerial photos taken by the government of Antioquia during 2012. 2012 Pre-event conditions comparison.

Soils description Physical description of the soils of the region done by (?) 2008 Hydrological model setup.

Table 2. Summary of the data used for the model setup.

$R_{i}$ is the vertical flow through tanks. The description of this variable is now included in the document and in the caption of Figure 4.

T11 Page 11, line 304 - 307: this information has to be provided at the end of the manuscript.

The information was moved to the acknowledgements section.

T12 Page 15-16: please revise all the symbol definition of the Hydroflash model.

The model description was revised and edited.

T13 Page 16, line 426: please define $A$ in the vicinity of it first occurrence

The paragraph was edited in order to give a proper representation of variable A.

Horizontal flow equations could be either linear or potential, as shown in equation

(1). In the modified hydrological model, $\beta$ and $\alpha$ are estimated by the user and 
then set into the model. From equations (1) to (3) $A_{i}(t)$ corresponds to the sectional area of each tank, $A_{i}(t)$ vary in function of the tank storage $S_{i}(t)$ (equation HESSD (4).

T14 Figure 7: the peak discharge interval used for validation should be indicated in this figure.

In the new version, the discharge interval use for validation is marked now with a red square.

T15 Figure 9 and 10: These figure 9 and 10 might be merged, presenting on the left side the 50 groups categorization (right panel of the figure 9), and the current figure 10 on the right side. The same key color defining the 50 groups categorization should be used in the map (Fig. 9) and to define somehow the related scale of the fig. 10.

In the Figure 3 we present how Figure 9 and 10 were merged in the revised document.

T16 Along all the manuscript, the unity has not to be in italic font. In addition, change the annotation $\mathrm{m} / \mathrm{s}$ to $\mathrm{m} . \mathrm{s}-1$.

The units were changed. 


\section{HESSD}

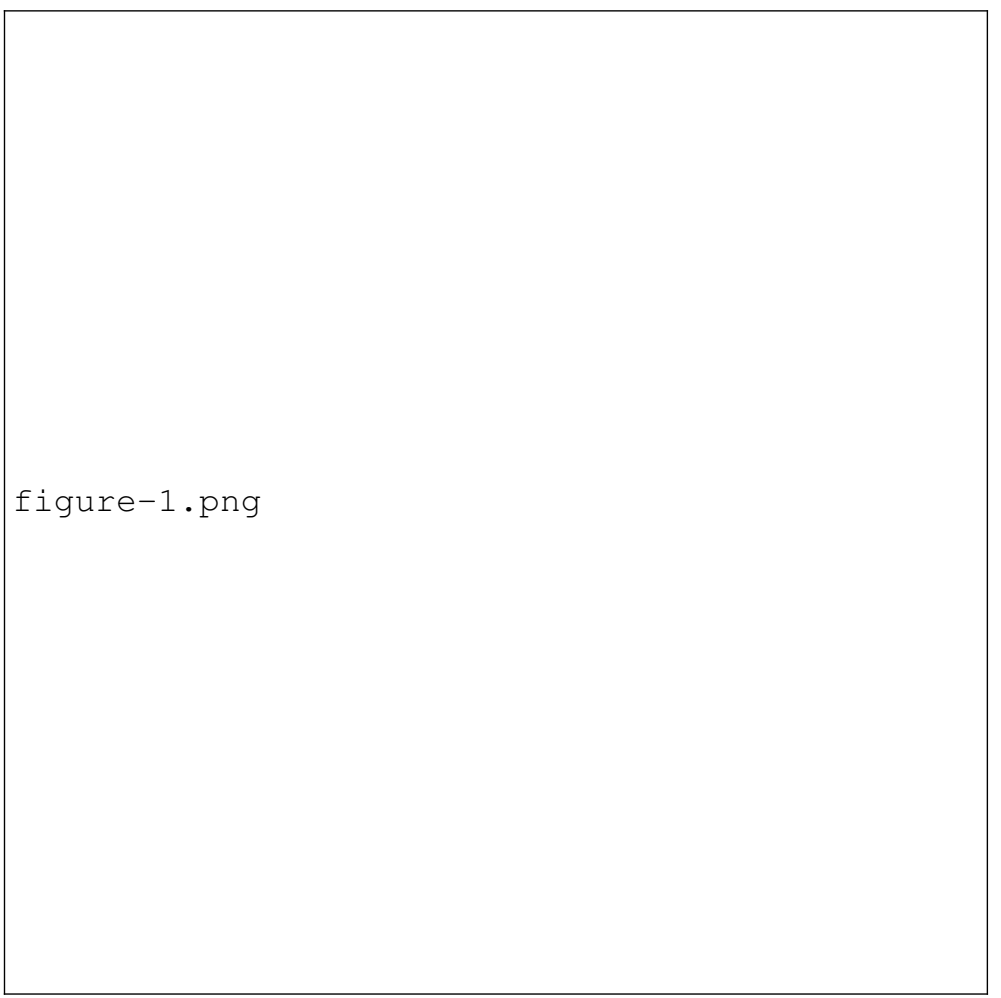

Interactive

comment

Fig. 3. Edited figure.

Printer-friendly version

Discussion paper

C18

(c) (i) 